To appear in Publ. Math. Debrecen

\title{
BERNSTEIN-DOETSCH TYPE THEOREMS FOR SET-VALUED MAPS OF STRONGLY AND APPROXIMATELY CONVEX AND CONCAVE TYPE
}

\author{
CARLOS GONZÁLEZ, KAZIMIERZ NIKODEM, ZSOLT PÁLES, AND GARI ROA \\ This paper is dedicated to the 90th birthday of Professor Lajos Tamássy.
}

\begin{abstract}
In this paper, we investigate properties of set-valued mappings that establish connection between the values of this map at two arbitrary points of the domain and the value at their midpoint. Such properties are, for instance, Jensen convexity/concavity, $K$-Jensen convexity/concavity (where $K$ is the set of nonnegative elements of an ordered vector space), and approximate/strong $K$-Jensen convexity/concavity. Assuming weak but natural regularity assumptions on the set-valued map, our main purpose is to deduce the convexity/concavity consequences of these properties in the appropriate sense. Our two main theorems will generalize most of the known results in this field, in particular the celebrated Bernstein-Doetsch Theorem from 1915, and thus they offer a unified view of these theories.
\end{abstract}

\section{INTRODUCTION}

The classical theorem of Bernstein and Doetsch [3] published almost one hundred years ago plays, undoubtedly, a fundamental role in the theory of convexity. This theorem asserts that if $f: D \rightarrow \mathbb{R}$ is a Jensen convex function (where $D$ is a real interval, or more generally, $D$ is a convex subset of a linear space $X$ ), i.e.,

$$
f\left(\frac{x+y}{2}\right) \leq \frac{f(x)+f(y)}{2} \quad(x, y \in D)
$$

and it is also locally upper bounded, then it must be convex over $D$. If $(-f)$ is Jensen convex, then $f$ is frequently called Jensen concave, the results for these functions are analogous under the assumption of local lower boundedness. The above theorem was seminal and was applied and generalized in many useful ways to various important circumstances that we briefly describe in what follows.

When the co-domain of $f$ is an ordered vector space $Y$, i.e., the set $K$ of the nonnegative elements in $Y$ forms a convex cone, then one can define $K$-Jensen convexity of $f: D \rightarrow Y$ by

$$
\frac{f(x)+f(y)}{2} \in f\left(\frac{x+y}{2}\right)+K \quad(x, y \in D) .
$$

In particular, if $Y=\mathbb{R}$ and $K=\mathbb{R}_{+}$, then (2) is equivalent to (11). Analogously, one can introduce $K$ convexity. Extensions of the Bernstein-Doetsch Theorem to this setting were formulated by Trudzik

Date: September 19, 2021.

2010 Mathematics Subject Classification. Primary 26B25. Secondary 54C60, 39B62.

Key words and phrases. K-Jensen convexity/concavity, set-valued map, Takagi transformation, approximate convexity, strong convexity.

This research of the third author was realized in the frames of TÁMOP 4.2.4. A/2-11-1-2012-0001 "National Excellence Program - Elaborating and operating an inland student and researcher personal support system". The project was subsidized by the European Union and co-financed by the European Social Fund. This research of the third author was also supported by the Hungarian Scientific Research Fund (OTKA) Grant NK 81402. 
[33]. Functions $f: D \rightarrow Y$ satisfying the inclusion

$$
f\left(\frac{x+y}{2}\right) \in \frac{f(x)+f(y)}{2}+K \quad(x, y \in D)
$$

are called $K$-Jensen concave. Obviously, this holds if and only if $(-f)$ is $K$-Jensen convex (or if $f$ is $(-K)$-Jensen convex). Thus, the results related to $K$-Jensen concavity of functions can always be derived from the statements established for $K$-Jensen convexity. (This, however, will not be the case for the set-valued setting.)

A further generalization step was to consider set-valued functions instead of single-valued ones. A set-valued map $F: D \rightarrow 2^{Y}$ is called $K$-Jensen convex if the inclusion

$$
\frac{F(x)+F(y)}{2} \subseteq F\left(\frac{x+y}{2}\right)+K \quad(x, y \in D)
$$

holds. Observe that if $F$ is of the form $F(x)=\{f(x)\}$ for some function $f: D \rightarrow Y$, then (4) is equivalent to (2), and hence (4) generalizes (2). Bernstein-Doetsch type results for this setting have been obtained by Averna, Cardinali, Nikodem, and Papalini [1, 6, 21, 22, 23, 24, 25] and by Borwein [5]. The notion of the $K$-Jensen concavity of a set-valued function $F: D \rightarrow 2^{Y}$, analogously to (3), is defined by

$$
F\left(\frac{x+y}{2}\right) \subseteq \frac{F(x)+F(y)}{2}+K \quad(x, y \in D) .
$$

Observe that, in general, the $K$-Jensen concavity of $F$ is not equivalent to the $K$-Jensen convexity of $(-F)$. Thus, starting from the set-valued setting, the cases of convexity and concavity need separate (but similar) considerations.

Another chain of generalizations of the Bernstein-Doetsch Theorem emerged from the paper of $\mathrm{Ng}$ and Nikodem [20] in the context of approximate convexity. They proved if $f: D \rightarrow \mathbb{R}$ is $\varepsilon$-Jensen convex for some $\varepsilon \geq 0$, i.e.,

$$
f\left(\frac{x+y}{2}\right) \leq \frac{f(x)+f(y)}{2}+\varepsilon \quad(x, y \in D)
$$

and if $f$ is also locally upper bounded, then it is $2 \varepsilon$-convex, i.e.,

$$
f(t x+(1-t) y) \leq t f(x)+(1-t) f(y)+2 \varepsilon \quad(x, y \in D, t \in[0,1]) .
$$

This result was also independently established by Laczkovich [13]. The set-valued and $K$-Jensen convex/concave variant of this theorem will be formulated as a consequence of our results in the sequel.

Considering more general error terms than constant ones, Házy and Páles [9] investigated the following approximate Jensen type inequality:

$$
f\left(\frac{x+y}{2}\right) \leq \frac{f(x)+f(y)}{2}+\varepsilon\|x-y\| \quad(x, y \in D)
$$

provided that $D$ is a subset of a normed space $X$ and $f$ is real valued. They showed, under the usual local upper-boundedness condition, that (8) implies

$$
f(t x+(1-t) y) \leq t f(x)+(1-t) f(y)+2 \varepsilon T(t)\|x-y\| \quad(x, y \in D, t \in[0,1]),
$$

where the function $T: \mathbb{R} \rightarrow \mathbb{R}$, the so-called Takagi function, is defined by

$$
T(t):=\sum_{n=0}^{\infty} \frac{1}{2^{n}} \operatorname{dist}\left(2^{n} t, \mathbb{Z}\right) .
$$


Results, extending this approach to more general error terms and also to convexity concepts related to Chebyshev systems, have recently been obtained by Házy, Makó and Páles [7, 8, 10, 11, 15, 16, 17, 18] and by Mureńko, Ja. Tabor, Jó. Tabor, and Żoldak [19, 29, 30, 31, 32]. For the set-valued and $K$-Jensen convex/concave setting more general statements will be formulated as direct consequences of our two main results below.

Finally, we mention the notion of strong convexity, which in some sense, is opposite to approximate convexity. Following Polyak [26], a function $f: D \rightarrow \mathbb{R}$ is called strongly Jensen convex with modulus $\varepsilon \geq 0$ if

$$
f\left(\frac{x+y}{2}\right) \leq \frac{f(x)+f(y)}{2}-\frac{\varepsilon}{4}\|x-y\|^{2} \quad(x, y \in D) .
$$

Assuming local upper boundedness of $f$, Azócar, Gimenez, Nikodem and Sanchez in [2] showed that the above strong Jensen-convexity property implies that $f$ is strongly convex with modulus $\varepsilon$, i.e.,

$$
f(t x+(1-t) y) \leq t f(x)+(1-t) f(y)-\varepsilon t(1-t)\|x-y\|^{2} \quad(x, y \in D, t \in[0,1]) .
$$

The set-valued Jensen convex variant of this result was established by Leiva, Merentes, Nikodem, and Sanchez [14]. More abstract and powerful results will be the corollaries of our two main theorems.

\section{Terminology and Auxiliary Results}

Let $X$ be a Hausdorff topological linear space. The family of all open sets containing the origin of $X$ will be denoted by $\mathcal{U}=\mathcal{U}(X)$. The closure of a subset $H \subseteq X$ will be denoted either by $\bar{H}$ or by $\operatorname{cl}(H)$. We will frequently use the fact that $\bar{H}=\bigcap_{U \in \mathcal{U}(X)}(U+H)$. An immediate consequence of this formula is that, for every pair of subsets $A, B \subseteq X$, we have

$$
\bar{A}+\bar{B} \subseteq \overline{A+B}=\overline{\bar{A}+\bar{B}} .
$$

(See, e.g., [27], [28]). Indeed, to prove the inclusion in (13), let $U \in \mathcal{U}(X)$ and choose $V \in \mathcal{U}(X)$ such that $V+V \subseteq U$. Then $\bar{A}+\bar{B} \subseteq(V+A)+(V+B) \subseteq U+A+B$. Hence $\bar{A}+\bar{B} \subseteq \bigcap_{U \in \mathcal{U}(X)}(U+A+B)=$ $\overline{A+B}$. The equality in (13) trivially follows from this inclusion and the monotonicity of the closure operation. If either $\bar{A}$ or $\bar{B}$ is compact then, the set $\bar{A}+\bar{B}$ is closed and the inclusion in (13) can be replaced by equality.

A subset $H \subseteq X$ is called bounded if, for any $U \in \mathcal{U}$, there exists $t>0$ such that $H \subseteq t U$. It is well-known that the union of finitely many bounded sets is also bounded. The family of bounded sets is closed under algebraic addition and multiplication by scalars.

Given two points $x, y \in X$, their convex hull, i.e., the segment connecting them, will be denoted by $[x, y]$. Convexity of a set $H \subseteq X$ is equivalent to the property that $[x, y] \subseteq H$ whenever $x, y \in H$. A set $H \subseteq X$ is said to be closedly convex if, for all $x, y \in H,[x, y] \subseteq \bar{H}$ holds. In fact, it is easy to see that $H$ is closedly convex if and only if $\bar{H}$ is convex. A convex set which is closed with respect to multiplication by positive scalars is called a convex cone. A set $H \subseteq X$ is called star-shaped with respect to a point $p \in H$, if for all $x \in H$, the segment $[x, p]$ is contained in $H$.

Given a convex cone $K \subseteq X$, we can define the ordering relation $\leq_{K}$ as follows: $x \leq_{K} y$ holds if and only if $y-x \in K$. This relation is transitive, and, provided that $K \cap(-K)=\{0\}$, it is also reflexive and antisymmetric and then $\leq_{K}$ is a partial order on $X$.

Given a convex cone $K$, a subset $S \subseteq X$ is called $K$-lower bounded if there exists a bounded set $H \subseteq X$ such that $S \subseteq H+K$. If $S \subseteq \operatorname{cl}(H+K)$ for some bounded set $H$, then we say that $S$ is closedly $K$-lower bounded. If the space $X$ is locally bounded, then these notions are equivalent to each other. One can easily see that the union of finitely many (closedly) $K$-lower bounded sets is also 
(closedly) $K$-lower bounded. Furthermore, the family of (closedly) $K$-lower bounded sets is closed under algebraic addition and multiplication by positive scalars.

A set $H \subseteq X$ is called $K$-convex (resp. closedly $K$-convex) if $[x, y]$ is contained in $H+K$ (resp. in $\operatorname{cl}(H+K)$ ) whenever $x, y \in H$. In general, using the inclusion in (13), one can see that the $\bar{K}$ convexity of $\bar{H}$ implies that $H$ is closedly $K$-convex, but the reversed implication may not be valid for all convex cones $K$.

Given a nonempty subset $H \subseteq X$, we can naturally attach a cone, called the recession cone of $H$ in the following manner:

$$
\operatorname{rec}(H):=\{x \in X \mid t x+H \subseteq H \text { for all } t \geq 0\} .
$$

It is elementary to check that $\operatorname{rec}(H)$ is a convex cone containing 0 . The additional basic properties of the recession cone are summarized in the following lemma.

Lemma 2.1. Let $H \subseteq X$ be a nonempty set. Then

(i) $\operatorname{rec}(H)$ is a convex cone containing 0 ;

(ii) $K=\operatorname{rec}(H)$ is the largest cone $K$ such that $K+H \subseteq H$ is valid;

(iii) $\overline{\operatorname{rec}}(H) \subseteq \operatorname{rec}(\bar{H})$;

(iv) for all $x \in X, t>0$, $\operatorname{rec}(x+t H)=\operatorname{rec}(H)$;

(v) for all nonempty sets $H_{1}, H_{2} \subseteq X$, $\operatorname{rec}\left(H_{1}\right)+\operatorname{rec}\left(H_{2}\right) \subseteq \operatorname{rec}\left(H_{1}+H_{2}\right)$.

Proof. The proofs of (i), (ii) and (iv) are immediate.

Taking closure of both sides of the inclusion $\operatorname{rec}(H)+H \subseteq H$ and then using (13), it follows that $\overline{\operatorname{rec}}(H)+\bar{H} \subseteq \bar{H}$. Hence, using (ii), we conclude that $\overline{\operatorname{rec}}(H) \subseteq \operatorname{rec}(\bar{H})$ proving (iii).

Adding up the relations $\operatorname{rec}\left(H_{1}\right)+H_{1} \subseteq H_{1}$ and $\operatorname{rec}\left(H_{2}\right)+H_{2} \subseteq H_{2}$ side by side, we get $\left(\operatorname{rec}\left(H_{1}\right)+\right.$ $\left.\operatorname{rec}\left(H_{2}\right)\right)+H_{1}+H_{2} \subseteq H_{1}+H_{2}$. Hence, using (ii), the inclusion stated in (v) follows.

In order to prove our main results in the next section, we will also need the following lemma, which allows one to perform the limit related to inclusions.

Lemma 2.2. Let $\left(A_{n}\right),\left(B_{n}\right)$ be nondecreasing sequences of nonempty subsets of $X$, let $H \subseteq X$ be a nonempty bounded set, let $K \subseteq \overline{\mathrm{rec}}\left(B_{0}\right)$ be also nonempty, and let $\varepsilon_{n}>0$ be a null-sequence of real numbers. Assume that, for all $n \geq 0$,

$$
A_{n} \subseteq \operatorname{cl}\left(\varepsilon_{n} H+K+B_{n}\right) .
$$

Then

$$
\operatorname{cl}\left(\bigcup_{n=0}^{\infty} A_{n}\right) \subseteq \operatorname{cl}\left(\bigcup_{n=0}^{\infty} B_{n}\right)
$$

Proof. Let $U \subseteq X$ be any neighborhood of the origin $0 \in X$ and let $V \subseteq X$ be an open balanced neighborhood of 0 such that $V+V+V \subseteq U$. The set $K$ being a subset of the closure of the recession cone $\operatorname{rec}\left(B_{n}\right)$, we have that $K \subseteq V+\operatorname{rec}\left(B_{n}\right)$. By definition, we also have that $\operatorname{rec}\left(B_{n}\right)+B_{n} \subseteq B_{n}$. Thus, for all $n \geq 0$, it follows that $K+B_{n} \subseteq V+B_{n}$.

On the other hand, since $H$ is bounded and $\varepsilon_{n}$ is a null-sequence, there exists an integer $N$ such that, for $n \geq N, \varepsilon_{n} H \subseteq V$. Therefore, applying (14), for $n \geq N$, we get that

$$
A_{n} \subseteq \mathrm{cl}\left(\varepsilon_{n} H+K+B_{n}\right) \subseteq \mathrm{cl}\left(V+V+B_{n}\right) \subseteq V+V+V+B_{n} \subseteq U+B_{n} \subseteq U+\bigcup_{k=0}^{\infty} B_{k} .
$$


Therefore,

$$
\bigcup_{n=N}^{\infty} A_{n} \subseteq U+\bigcup_{k=0}^{\infty} B_{k}
$$

In view of the nondecreasingness of the sequence $\left(A_{n}\right)$, inclusion (16) is equivalent to

$$
\bigcup_{n=0}^{\infty} A_{n} \subseteq U+\bigcup_{k=0}^{\infty} B_{k}
$$

The above relation is valid for all $U \in \mathcal{U}(X)$, hence

$$
\bigcup_{n=0}^{\infty} A_{n} \subseteq \bigcap_{U \in \mathcal{U}(X)}\left(U+\bigcup_{k=0}^{\infty} B_{k}\right)=\mathrm{cl}\left(\bigcup_{k=0}^{\infty} B_{k}\right) .
$$

The right hand side of the above inclusion is closed, therefore (15) follows immediately.

Given another Hausdorff topological linear space $Y$, we will now introduce further notions for setvalued maps from a subset of $X$ to $\mathcal{P}_{0}(Y)$, where $\mathcal{P}_{0}(Y)$ stands for the family of nonempty subsets of the space $Y$.

Let $D \subseteq X$ be a nonempty set and $K$ be a convex cone in $Y$. A set-valued function $S: D \rightarrow \mathcal{P}_{0}(Y)$ is called locally closedly $K$-lower bounded if, for any $x \in D$, there exist an open set $U$ containing $x$ and a bounded set $H \subseteq Y$ such that $S(u) \subseteq \operatorname{cl}(H+K)$ holds for all $u \in U \cap D$. A set-valued function $S: D \rightarrow \mathcal{P}_{0}(Y)$ is called locally closedly weakly $K$-upper bounded if, for any $x \in D$, there exist an open set $U$ containing $x$ and a bounded set $H \subseteq Y$ such that $0 \in \operatorname{cl}(S(u)+H+K)$ for all $u \in U \cap D$.

Lemma 2.3. Assume that $S: D \rightarrow \mathcal{P}_{0}(Y)$ is locally closedly $K$-lower bounded set-valued map. Then, for each compact subset $C \subseteq D$, there exists a bounded set $H \subseteq Y$ such that, for all $x \in C$, $S(x) \subseteq \operatorname{cl}(H+K)$.

Proof. Let $C \subseteq D$ be a nonempty compact set. Since $S$ is locally closedly $K$-lower bounded, therefore, for each $x \in C$, there exist an open set $U_{x}$ containing $x$ and a bounded set $H_{x} \subseteq Y$ such that $S(u) \subseteq \operatorname{cl}\left(H_{x}+K\right)$ for all $u \in U_{x} \cap D$. The family of sets $\left\{U_{x} \mid x \in C\right\}$ is an open covering for $C$, hence, by the compactness of $C$, there exists a finite subcovering $\left\{U_{x_{1}}, \ldots, U_{x_{n}}\right\}$ of $C$. Let $H:=H_{x_{1}} \cup \cdots \cup H_{x_{n}}$. Then, $H$ is bounded and, for all $x \in C$, there exists $i \in\{1, \ldots, n\}$ such that $x \in U_{x_{i}}$. Hence $S(x) \subseteq \operatorname{cl}\left(H_{x_{i}}+K\right) \subseteq \operatorname{cl}(H+K)$, which proves the assertion.

Lemma 2.4. Assume that $S: D \rightarrow \mathcal{P}_{0}(Y)$ is locally closedly weakly $K$-upper bounded set-valued map. Then, for each compact subset $C \subseteq D$, there exists a bounded set $H \subseteq Y$ such that, for all $x \in C$, $0 \in \operatorname{cl}(S(x)+H+K)$.

Proof. Let $C \subseteq D$ be a nonempty compact set. The set-valued map $S$ being locally closedly weakly $K$ upper bounded, it follows that, for each $x \in C$, there exist an open set $U_{x}$ containing $x$ and a bounded set $H_{x} \subseteq Y$ such that $0 \in \operatorname{cl}\left(S(u)+H_{x}+K\right)$ for all $u \in U_{x}$. The family of sets $\left\{U_{x} \mid x \in C\right\}$ is an open covering for $C$, hence, by the compactness of $C$, there exists a finite subcovering $\left\{U_{x_{1}}, \ldots, U_{x_{n}}\right\}$ of $C$. Let $H:=H_{x_{1}} \cup \cdots \cup H_{x_{n}}$. Then, $H$ is bounded and, for all $x \in C$, there exists $i \in\{1, \ldots, n\}$ such that $x \in U_{x_{i}}$. Hence $0 \in \operatorname{cl}\left(S(x)+H_{x_{i}}+K\right) \subseteq \operatorname{cl}(S(x)+H+K)$, which proves the assertion. 


\section{TAKAGI TRANSFORMATION OF SET-VALUED MAPS}

Assume now that $D \subseteq X$ is a star-shaped set and consider now a set-valued map $S: D \rightarrow \mathcal{P}_{0}(Y)$ with the additional property that $0 \in S(x)$ for all $x \in D$. For such a map, we define $S^{T}: \mathbb{R} \times D \rightarrow Y$ by the following expression:

$$
S^{T}(t, x):=\operatorname{cl}\left(\bigcup_{n=0}^{\infty} \sum_{k=0}^{n} \frac{1}{2^{k}} S\left(2 d_{\mathbb{Z}}\left(2^{k} t\right) x\right)\right) \quad(t \in \mathbb{R}, x \in D),
$$

where $d_{\mathbb{Z}}: \mathbb{R} \rightarrow \mathbb{R}$ is defined by

$$
d_{\mathbb{Z}}(t):=\operatorname{dist}(t, \mathbb{Z}):=\inf _{k \in \mathbb{Z}}|k-t| \quad(t \in \mathbb{R}) .
$$

The set-valued map $S^{T}$ will be called the Takagi transformation of the map $S$ in the sequel. The recession cone of a set-valued map $S$ is set by

$$
\operatorname{rec}(S):=\bigcap_{x \in D} \operatorname{rec} S(x)
$$

In the following lemma we establish the relationship between a set-valued map and its Takagi transformation.

Lemma 3.5. Let $D \subseteq X$ be a star-shaped set and $S: D \rightarrow \mathcal{P}_{0}(Y)$ be a set-valued map with the additional property that $0 \in S(x)$ for all $x \in D$. Then

$$
\operatorname{cl}(S(x)) \subseteq S^{T}\left(\frac{1}{2}, x\right) \quad(x \in D) .
$$

If, in addition $S(0) \subseteq \overline{\operatorname{rec}}(S)$, then

$$
\operatorname{cl}(S(x))=S^{T}\left(\frac{1}{2}, x\right) \quad(x \in D) .
$$

Proof. Observe that $d_{\mathbb{Z}}\left(\frac{1}{2}\right)=\frac{1}{2}$ and $d_{\mathbb{Z}}\left(2^{k} \cdot \frac{1}{2}\right)=0$ for $k \in \mathbb{N}$. Thus,

$$
S^{T}\left(\frac{1}{2}, x\right)=\operatorname{cl}\left(\bigcup_{n=0}^{\infty} \sum_{k=0}^{n} \frac{1}{2^{k}} S\left(2 d_{\mathbb{Z}}\left(2^{k} \cdot \frac{1}{2}\right) x\right)\right)=\mathrm{cl}\left(S(x)+\bigcup_{n=0}^{\infty} \sum_{k=1}^{n} \frac{1}{2^{k}} S(0)\right) .
$$

The property $0 \in S(0)$, directly implies that (18) holds. To prove (19), assume that $S(0) \subseteq \overline{\operatorname{rec}}(S)$. Then $S(0) \subseteq \overline{\operatorname{rec}}(S(x)) \subseteq \operatorname{rec}(\overline{S(x)})$. Since $\operatorname{rec}(\overline{S(x)})$ is a convex cone, we have that this set is closed under addition and multiplication by scalars. Thus, for all $n \in \mathbb{N}$,

$$
\sum_{k=1}^{n} \frac{1}{2^{k}} S(0) \subseteq \sum_{k=1}^{n} \frac{1}{2^{k}} \operatorname{rec}(\overline{S(x)}) \subseteq \operatorname{rec}(\overline{S(x)}) .
$$

Consequently,

$$
\bigcup_{n=0}^{\infty} \sum_{k=1}^{n} \frac{1}{2^{k}} S(0) \subseteq \operatorname{rec}(\overline{S(x)})
$$

Therefore,

$$
S^{T}\left(\frac{1}{2}, x\right)=\operatorname{cl}\left(S(x)+\bigcup_{n=0}^{\infty} \sum_{k=1}^{n} \frac{1}{2^{k}} S(0)\right) \subseteq \operatorname{cl}(\overline{S(x)}+\operatorname{rec}(\overline{S(x)})) \subseteq \operatorname{cl}(S(x)),
$$

which completes the proof of (19). 
The following lemma implies that the Takagi transformation of a set-valued map which is constructed as the product of an upper semicontinuous nonnegative scalar function and a convex subset of $Y$ is the product of the Takagi transformation of the scalar function and the same set.

Proposition 3.6. Let $D \subseteq X$ be a star-shaped set and $S_{0} \subseteq Y$ be a convex set containing $0 \in Y$ and $K \subseteq Y$ be a convex cone. Let $\varphi: D \rightarrow \mathbb{R}_{+}$be a locally upper bounded nonnegative function. Define $S: D \rightarrow \mathcal{P}_{0}(Y)$ by $S(x):=K+\varphi(x) S_{0}$. Then

$$
S^{T}(t, x)=\operatorname{cl}\left(K+\varphi^{T}(t, x) S_{0}\right) \quad(t \in \mathbb{R}, x \in D),
$$

where

$$
\varphi^{T}(t, x)=\sum_{n=0}^{\infty} \frac{1}{2^{n}} \varphi\left(2 d_{\mathbb{Z}}\left(2^{n} t\right) x\right) \quad(t \in \mathbb{R}, x \in D) .
$$

If, in addition, $\varphi(0)=0$, then

$$
\varphi^{T}\left(\frac{1}{2}, x\right)=\varphi(x) \quad \text { and } \quad S^{T}\left(\frac{1}{2}, x\right)=\operatorname{cl}\left(K+\varphi(x) S_{0}\right)=\operatorname{cl}(S(x)) \quad(x \in D) .
$$

Proof. For $t \in \mathbb{R}$ and $n \geq 0$, we have that $0 \leq 2 d_{\mathbb{Z}}\left(2^{n} t\right) \leq 1$, therefore $2 d_{\mathbb{Z}}\left(2^{n} t\right) x \in[0, x]$. The function $\varphi$ being locally upper bounded on $D$, it is bounded from above over $[0, x]$ by some constant $M(x)$. Then we have that

$$
\varphi^{T}(t, x)=\sum_{n=0}^{\infty} \frac{1}{2^{n}} \varphi\left(2 d_{\mathbb{Z}}\left(2^{n} t\right) x\right) \leq \sum_{n=0}^{\infty} \frac{1}{2^{n}} M(x)=2 M(x) \quad(t \in \mathbb{R}) .
$$

To prove (201), fix $(t, x) \in \mathbb{R} \times D$.

For the proof of the inclusion $\subseteq$ in (20), we first show that

$$
\bigcup_{n=0}^{\infty} \sum_{k=0}^{n} \frac{1}{2^{k}} S\left(2 d_{\mathbb{Z}}\left(2^{k} t\right) x\right) \subseteq K+\varphi^{T}(t, x) S_{0} .
$$

Choose $y$ from the left hand side of inclusion (23) arbitrarily. Then, using the definition of $S$ and the convexity of $S_{0}$, for some $n \geq 0$ we have that

$$
\begin{aligned}
y & \in \sum_{k=0}^{n} \frac{1}{2^{k}} S\left(2 d_{\mathbb{Z}}\left(2^{k} t\right) x\right)=\sum_{k=0}^{n}\left(K+\frac{1}{2^{k}} \varphi\left(2 d_{\mathbb{Z}}\left(2^{k} t\right) x\right) S_{0}\right) \\
& =K+\left(\sum_{k=0}^{n} \frac{1}{2^{k}} \varphi\left(2 d_{\mathbb{Z}}\left(2^{k} t\right) x\right)\right) S_{0}+\left(\sum_{k=n+1}^{\infty} \frac{1}{2^{k}} \varphi\left(2 d_{\mathbb{Z}}\left(2^{k} t\right) x\right)\right)\{0\} \\
& \subseteq K+\left(\sum_{k=0}^{\infty} \frac{1}{2^{k}} \varphi\left(2 d_{\mathbb{Z}}\left(2^{k} t\right) x\right)\right) S_{0}=K+\varphi^{T}(t, x) S_{0} .
\end{aligned}
$$

Thus, inclusion (23) is proved. Taking the closures of both sided, the inclusion $\subseteq$ in (20) follows immediately.

For the proof of the inclusion $\supseteq$ in (20), by the closedness of $S^{T}(t, x)$, it suffices to show that

$$
K+\varphi^{T}(t, x) S_{0} \subseteq S^{T}(t, x) .
$$

Let $y \in K+\varphi^{T}(t, x) S_{0}$. Then $y=u+\varphi^{T}(t, x) v$ for some elements $u \in K, v \in S_{0}$. Define the sequence $\left(y_{n}\right)$ by

$$
y_{n}:=u+\sum_{k=0}^{n} \frac{1}{2^{k}} \varphi\left(2 d_{\mathbb{Z}}\left(2^{k} t\right) x\right) v .
$$


Obviously, $y_{n} \rightarrow y$ as $n \rightarrow \infty$. On the other hand, for all $n \geq 0$

$$
\begin{aligned}
y_{n} \in K+\sum_{k=0}^{n} \frac{1}{2^{k}} \varphi\left(2 d_{\mathbb{Z}}\left(2^{k} t\right) x\right) S_{0} & =\sum_{k=0}^{n} \frac{1}{2^{k}}\left(K+\varphi\left(2 d_{\mathbb{Z}}\left(2^{k} t\right) x\right) S_{0}\right) \\
& =\sum_{k=0}^{n} \frac{1}{2^{k}} S\left(2 d_{\mathbb{Z}}\left(2^{k} t\right) x\right) \subseteq S^{T}(t, x) .
\end{aligned}
$$

By the closedness of $S^{T}(t, x)$, it follows that the limit $y$ of the sequence $\left(y_{n}\right)$ is also contained in $S^{T}(t, x)$, which completes the proof of (20) .

In the case of $\varphi(0)=0$, the first equality in (22) is immediate, the second equality is then a consequence of (20) .

Corollary 3.7. Let $X$ be a normed space, $D \subseteq X$ be a star-shaped set, $S_{0} \subseteq Y$ be a convex set containing $0 \in Y, K \subseteq Y$ be a convex cone, and $\alpha>0$. Define $S: D \rightarrow \mathcal{P}_{0}(Y)$ by $S(x):=K+\|x\|^{\alpha} S_{0}$. Then

$$
S^{T}(t, x)=\operatorname{cl}\left(K+T_{\alpha}(t)\|x\|^{\alpha} S_{0}\right) \quad(t \in \mathbb{R}, x \in D),
$$

where $T_{\alpha}: \mathbb{R} \rightarrow \mathbb{R}$ is the $\alpha$-order Takagi type function defined by

$$
T_{\alpha}(t):=\sum_{n=0}^{\infty} 2^{\alpha-n}\left(d_{\mathbb{Z}}\left(2^{n} t\right)\right)^{\alpha} \quad(t \in \mathbb{R}) .
$$

Proof. To obtain the statement, we can apply Proposition 3.6 with the function $\varphi$ defined by $\varphi(x):=$ $\|x\|^{\alpha}$. Observe that

$$
\varphi^{T}(t, x)=\sum_{n=0}^{\infty} \frac{1}{2^{n}} \varphi\left(2 d_{\mathbb{Z}}\left(2^{n} t\right) x\right)=\sum_{n=0}^{\infty} 2^{\alpha-n}\left(d_{\mathbb{Z}}\left(2^{n} t\right)\right)^{\alpha}\|x\|^{\alpha}=T_{\alpha}(t)\|x\|^{\alpha} \quad(t \in \mathbb{R}, x \in D) .
$$

Therefore, (24) is a consequence of (20) of Proposition 3.6.

Remark 3.8. An important particular case is when $\alpha=1$, then $T_{1}=2 T$, where $T$ is the Takagi function defined by (10) in the introduction. In the case $\alpha=2$ an interesting argument results in a closed form for $T_{2}$. Observe that $T_{\alpha}$ (for any $\alpha>0$ ) satisfies the functional equation

$$
T_{\alpha}(t)=2^{\alpha}\left(d_{\mathbb{Z}}(t)\right)^{\alpha}+\frac{1}{2} T_{\alpha}(2 t) \quad(t \in \mathbb{R}) .
$$

By Banach's fixed-point theorem, this functional equation has a unique solution in the Banach space of bounded real functions over the real line (which is equipped with the supremum norm). Thus $T_{\alpha}$ is a unique solution to (26) . On the other hand, for $\alpha=2$, one can easily check that the 1-periodic function $T_{2}^{*}$ defined on $[0,1]$ by $T_{2}^{*}(t)=4 t(1-t)$ is also a solution of (26) , thus we must have $T_{2}(t)=4 t(1-t)$ for $t \in[0,1]$. For further details, see [18].

Corollary 3.9. Let $X$ be a normed space, $D \subseteq X$ be a star-shaped set, $S_{0} \subseteq Y$ be a convex set containing $0 \in Y, K \subseteq Y$ be a convex cone. Define $S: D \rightarrow \mathcal{P}_{0}(Y)$ by $S(x):=K+S_{0}$. Then

$$
S^{T}(t, x)=\operatorname{cl}\left(K+2 S_{0}\right) \quad(t \in \mathbb{R}, x \in D) .
$$

Proof. We apply Proposition [3.6 for the constant function $\varphi \equiv 1$. Then (21) yields that $\varphi^{T} \equiv 2$, whence (20) implies the statement. 


\section{Main Results}

The main results of this paper are contained in the following two theorems. Throughout this section, we assume that $X$ and $Y$ are Hausdorff topological linear spaces.

Theorem 4.1. Let $D \subseteq X$ be a nonempty convex set and $A, B:(D-D) \rightarrow \mathcal{P}_{0}(Y)$ such that $0 \in A(x) \cap B(x)$ for all $x \in(D-D)$. Denote $\overline{\mathrm{rec}}(B)$, the closure of the recession cone of $B$ by $K$. Let $F: D \rightarrow \mathcal{P}_{0}(Y)$ be a set-valued mapping which satisfies the Jensen-convexity-type inclusion

$$
\frac{F(x)+F(y)}{2}+A(x-y) \subseteq \operatorname{cl}\left(F\left(\frac{x+y}{2}\right)+B(x-y)\right) \quad(x, y \in D) .
$$

Assume, in addition that $F$ has the following two K-boundedness properties.

(i) $F$ is pointwise closedly $K$-lower bounded, i.e., for each $x \in D$, there exists a bounded set $H \subseteq Y$ such that $F(x) \subseteq \operatorname{cl}(H+K)$;

(ii) $F$ is locally closedly weakly $K$-upper bounded on $D$, i.e., for all $x \in D$, there exist an open set $U$ containing $x$ and a bounded set $H \subseteq Y$ such that $0 \in \operatorname{cl}(F(u)+H+K)$ holds for all $u \in U$.

Then $F$ satisfies the convexity type inclusion

$$
t F(x)+(1-t) F(y)+A^{T}(t, x-y) \subseteq \operatorname{cl}\left(F(t x+(1-t) y)+B^{T}(t, x-y)\right) \quad(x, y \in D, t \in[0,1]) .
$$

Theorem 4.2. Let $D \subseteq X$ be a nonempty convex set and $A, B:(D-D) \rightarrow \mathcal{P}_{0}(Y)$ such that $0 \in A(x) \cap B(x)$ for all $x \in(D-D)$. Denote $\overline{\mathrm{rec}}(B)$, the closure of the recession cone of $B$ by $K$. Let $F: D \rightarrow \mathcal{P}_{0}(Y)$ be a set-valued mapping which satisfies the following Jensen-concavity-type inclusion

$$
F\left(\frac{x+y}{2}\right)+A(x-y) \subseteq \operatorname{cl}\left(\frac{F(x)+F(y)}{2}+B(x-y)\right) \quad(x, y \in D) .
$$

Assume, in addition that $F$ has the following $K$-convexity and $K$-boundedness properties.

(i) $F$ is pointwise closedly $K$-convex, i.e., $t F(x)+(1-t) F(x) \subseteq \operatorname{cl}(F(x)+K)$ holds for each $x \in D$ and for all $t \in[0,1]$;

(ii) $F$ is locally closedly $K$-lower bounded, i.e., for each $x \in D$, there exist a neighborhood $U$ of $x$ and a bounded set $H \subseteq Y$ such that $F(u) \subseteq \operatorname{cl}(H+K)$ for all $u \in D \cap U$.

Then $F$ satisfies the concavity type inclusion

$$
F(t x+(1-t) y)+A^{T}(t, x-y) \subseteq \mathrm{cl}\left(t F(x)+(1-t) F(y)+B^{T}(t, x-y)\right) \quad(x, y \in D, t \in[0,1])
$$

Remark 4.3. In each of the above theorems the closure operation can be removed from the right hand sides of the inclusions (28), (29), (30), and (31) if the values of the set-valued map $F$ are compact and $B$ has closed values. The closure operation can also be removed from the right hand sides of (28) and (29) if $F$ has closed values and $B$ is compact valued. This observation also applies to the corollaries below. Another thing is which is worth mentioning is that if $A(0) \subseteq \overline{\operatorname{rec}}(A)$ and $B(0) \subseteq \overline{\operatorname{rec}}(B)$, then, in view of Lemma 3.5, the inclusions (31) and (29) reduce to (30) and (28) for the substitution $t=\frac{1}{2}$, respectively. Therefore, in this case, under the boundedness and convexity assumptions on $F$, (31) and (29) are equivalent to (30) and (28), respectively. The problem whether inclusions (31) and (29) are the sharpest possible is an open problem. Results where the exactness of such estimates were obtained are due to Boros [4, Makó and Páles [15, 18]. 
The proofs of the above two theorems are described in the next section. In what follows, taking particular choices of the set-valued maps $A, B$ and using Proposition 3.6, we establish some of the important direct consequences of these theorems. They will illustrate how the results recalled in the introduction are related to our main theorems.

In the next four corollaries we suppose that $D \subseteq X$ is a nonempty convex set, $K \subseteq Y$ is a nonempty closed convex cone, $S_{0} \subseteq Y$ is a convex set containing 0 and $\varphi:(D-D) \rightarrow \mathbb{R}_{+}$is a locally upper bounded nonnegative function. Note that, by the convexity of $D$, the set $(D-D)$ is starshaped, thus Proposition 3.6 can be applied.

The first two corollaries are about approximately and strongly $K$-Jensen convex set-valued mapping, respectively.

Corollary 4.4. Assume that $F: D \rightarrow \mathcal{P}_{0}(Y)$ is a pointwise closedly $K$-lower bounded and locally closedly weakly $K$-upper bounded set-valued mapping which satisfies

$$
\frac{F(x)+F(y)}{2} \subseteq \mathrm{cl}\left(F\left(\frac{x+y}{2}\right)+K+\varphi(x-y) S_{0}\right) \quad(x, y \in D) .
$$

Then

$$
t F(x)+(1-t) F(y) \subseteq \operatorname{cl}\left(F(t x+(1-t) y)+K+\varphi^{T}(t, x-y) S_{0}\right) \quad(x, y \in D, t \in[0,1]) .
$$

Corollary 4.5. Assume that $F: D \rightarrow \mathcal{P}_{0}(Y)$ is a pointwise closedly $K$-lower bounded and locally closedly weakly $K$-upper bounded set-valued mapping which satisfies

$$
\frac{F(x)+F(y)}{2}+\varphi(x-y) S_{0} \subseteq \mathrm{cl}\left(F\left(\frac{x+y}{2}\right)+K\right) \quad(x, y \in D) .
$$

Then

$$
t F(x)+(1-t) F(y)+\varphi^{T}(t, x-y) S_{0} \subseteq \mathrm{cl}(F(t x+(1-t) y)+K) \quad(x, y \in D, t \in[0,1]) .
$$

The next two corollaries are about approximately and strongly $K$-Jensen concave set-valued mapping, respectively.

Corollary 4.6. Assume that $F: D \rightarrow \mathcal{P}_{0}(Y)$ is a pointwise closedly $K$-convex and locally closedly $K$-lower bounded set-valued mapping which satisfies

$$
F\left(\frac{x+y}{2}\right) \subseteq \operatorname{cl}\left(\frac{F(x)+F(y)}{2}+K+\varphi(x-y) S_{0}\right) \quad(x, y \in D) .
$$

Then

$$
F(t x+(1-t) y) \subseteq \operatorname{cl}\left(t F(x)+(1-t) F(y)+K+\varphi^{T}(t, x-y) S_{0}\right) \quad(x, y \in D, t \in[0,1]) .
$$

Corollary 4.7. Assume that $F: D \rightarrow \mathcal{P}_{0}(Y)$ is a pointwise closedly $K$-convex and locally closedly $K$-lower bounded set-valued mapping which satisfies

$$
F\left(\frac{x+y}{2}\right)+\varphi(x-y) S_{0} \subseteq \mathrm{cl}\left(\frac{F(x)+F(y)}{2}+K\right) \quad(x, y \in D) .
$$

Then

$$
F(t x+(1-t) y)+\varphi^{T}(t, x-y) S_{0} \subseteq \operatorname{cl}(t F(x)+(1-t) F(y)+K) \quad(x, y \in D, t \in[0,1]) .
$$


Proof of the Corollaries 4.4 4.7. Using Theorem 4.1 with the set-valued maps $A(u)=0$ and $B(u)=$ $K+\varphi(u) S_{0}$ (resp., $A(u)=\varphi(u) S_{0}$ and $B(u)=K$ ) and applying the Proposition 3.6, we obtain Corollary 4.4 (resp., Corollary 4.5). Observe that, in both settings, we have that $K \subseteq \overline{\operatorname{rec}}(B)$, thus the pointwise closed $K$-lower boundedness and local closed weak $K$-upper boundedness of $F$ imply its pointwise closed $\overline{\mathrm{rec}}(B)$-lower boundedness and local closed weak $\overline{\operatorname{rec}}(B)$-upper boundedness.

Analogously, using Theorem 4.2 with the set-valued maps $A(u)=0$ and $B(u)=K+\varphi(u) S_{0}$ (resp., $A(u)=\varphi(u) S_{0}$ and $B(u)=K$ ) and applying the Proposition [3.6, we obtain Corollary 4.6 (resp., Corollary 4.7). In both settings, we have that $K \subseteq \overline{\operatorname{rec}}(B)$, thus the pointwise closed $K$-convexity and local closed $K$-lower boundedness of $F$ imply its pointwise closed $\overline{\operatorname{rec}}(B)$-convexity and local closed $\overline{\mathrm{rec}}(B)$-lower boundedness.

Remark 4.8. The results mentioned and recalled in the introduction can be derived as obvious consequences of the above corollaries. In the real valued setting, the Bernstein-Doetsch Theorem 3 , the results of $\mathrm{Ng}-$ Nikodem [20] and Házy-Páles [9] follow if, in Corollary 4.4, we take $Y:=\mathbb{R}, K:=\mathbb{R}_{+}$, $S_{0}:=[-1,0], F(x):=\{f(x)\}$, and $\varphi(x):=0, \varphi(x):=\varepsilon, \varphi(x):=\varepsilon\|x\|$, respectively. Observe that, in these cases, Proposition [3.6 yields $\varphi^{T}(t, x):=0, \varphi^{T}(t, x):=2 \varepsilon, \varphi^{T}(t, x):=2 \varepsilon T(t)\|x\|$, respectively. The results of Averna, Cardinali, Nikodem, and Papalini [1, 6, 21, 22, 23, 24, 25] and by Borwein [5] that are related to $K$-Jensen convex/concave vector-valued and set-valued mappings can also be obtained directly. Numerous results obtained for approximate midconvexity by Makó and Páles [15, 18] and by Mureńko, Ja. Tabor, Jó. Tabor, and Żoldak [19, 29, 30, 31, 32, are generalized by Corollaries 4.4 4.7 to the vector-valued and set-valued setting. Similarly, using the explicit form of the function $T_{2}$ described in Remark 3.8, one can easily derive the results of Azócar, Gimenez, Nikodem and Sanchez [2] and Leiva, Merentes, Nikodem, and Sanchez [14] that are related to strongly $K$-Jensen convex real valued and set-valued functions from Corollary 4.5.

\section{Proofs of the Two Main Theorems}

Proof of Theorem 4.1. As the first step of the proof of (29), we are going to show that, for all $x, y \in D$ there exists a bounded set $H \subseteq Y$ such that, for all $n \geq 0, t \in[0,1]$,

$$
\begin{aligned}
t F(x)+(1-t) F(y) & +\sum_{k=0}^{n-1} \frac{1}{2^{k}} A\left(2 d_{\mathbb{Z}}\left(2^{k} t\right)(x-y)\right) \\
& \subseteq \mathrm{cl}\left(F(t x+(1-t) y)+\frac{1}{2^{n}} H+K+\sum_{k=0}^{n-1} \frac{1}{2^{k}} B\left(2 d_{\mathbb{Z}}\left(2^{k} t\right)(x-y)\right)\right) .
\end{aligned}
$$

Fix $x, y \in D$ arbitrarily. To verify that (40) holds, we will proceed by induction on $n$. For the case $n=0$, we have to prove that there exists a bounded set $H$ such that, for all $t \in[0,1]$,

$$
t F(x)+(1-t) F(y) \subseteq \mathrm{cl}(F(t x+(1-t) y)+H+K) .
$$

Let $U \in \mathcal{U}(Y)$ and choose a balanced $V \in \mathcal{U}(Y)$ such that $V+V+V \subseteq U$. Because $F$ is pointwise closedly $K$-lower bounded, there exist bounded sets $H_{x}, H_{y} \subseteq Y$ such that

$$
F(x) \subseteq \operatorname{cl}\left(H_{x}+K\right) \subseteq V+H_{x}+K \quad \text { and } \quad F(y) \subseteq \operatorname{cl}\left(H_{y}+K\right) \subseteq V+H_{y}+K .
$$

Multiplying these inclusions by $t$ and $1-t$, respectively, adding them up side by side, and using the convexity of $K$, we obtain

$$
\begin{aligned}
t F(x)+(1-t) F(y) & \subseteq t V+t H_{x}+t K+(1-t) V+(1-t) H_{y}+(1-t) K \\
& \subseteq V+V+t H_{x}+(1-t) H_{y}+K .
\end{aligned}
$$


One can prove that the sets $H_{1}:=\bigcup_{t \in[0,1]} t H_{x}$ and $H_{2}:=\bigcup_{t \in[0,1]}(1-t) H_{y}$ are bounded. Thus, inclusion (42) yields that, for all $t \in[0,1]$,

$$
t F(x)+(1-t) F(y) \subseteq V+V+H_{1}+H_{2}+K .
$$

On the other hand, applying Lemma 2.4, by the local closed weak $K$-upper boundedness and the compactness of the segment $[x, y]$, there exist a bounded set $H_{0}$ such that, for all $t \in[0,1]$,

$$
0 \in \operatorname{cl}\left(F(t x+(1-t) y)+H_{0}+K\right) \subseteq V+F(t x+(1-t) y)+H_{0}+K
$$

Now adding up the inclusions (43) and (44) side by side, for all $t \in[0,1]$, we obtain

$$
\begin{aligned}
t F(x)+(1-t) F(y) & \subseteq V+V+V+F(t x+(1-t) y)+H_{0}+H_{1}+H_{2}+K \\
& \subseteq U+F(t x+(1-t) y)+H_{0}+H_{1}+H_{2}+K .
\end{aligned}
$$

Therefore,

$$
\begin{aligned}
t F(x)+(1-t) F(y) & \subseteq \bigcap_{U \in \mathcal{U}}\left(U+F(t x+(1-t) y)+H_{0}+H_{1}+H_{2}+K\right) \\
& =\operatorname{cl}\left(F(t x+(1-t) y)+H_{0}+H_{1}+H_{2}+K\right) .
\end{aligned}
$$

Thus, inclusion (41) follows with $H:=H_{0}+H_{1}+H_{2}$.

Now, suppose that the inclusion (40) holds for $n$ and let us prove that it is also valid for $n+1$. Assume that $t \in\left[0, \frac{1}{2}\right]$ (the case when $t \in\left[\frac{1}{2}, 1\right]$ is completely analogous). Then $d_{\mathbb{Z}}(t)=t$ and we can write the left hand side of the inclusion as

$$
\begin{aligned}
t F(x) & +(1-t) F(y)+\sum_{k=0}^{n} \frac{1}{2^{k}} A\left(2 d_{\mathbb{Z}}\left(2^{k} t\right)(x-y)\right) \\
& =t F(x)+(1-t) F(y)+A(2 t(x-y))+\sum_{k=1}^{n} \frac{1}{2^{k}} A\left(2 d_{\mathbb{Z}}\left(2^{k} t\right)(x-y)\right) .
\end{aligned}
$$

We have that

$$
(1-t) F(y) \subseteq \frac{1-2 t}{2} F(y)+\frac{1}{2} F(y)
$$

and therefore

$$
\begin{aligned}
& t F(x)+(1-t) F(y)+A(2 t(x-y))+\sum_{k=1}^{n} \frac{1}{2^{k}} A\left(2 d_{\mathbb{Z}}\left(2^{k} t\right)(x-y)\right) \\
& \subseteq \frac{1}{2}\left(2 t F(x)+(1-2 t) F(y)+\sum_{k=0}^{n-1} \frac{1}{2^{k}} A\left(2 d_{\mathbb{Z}}\left(2^{k}(2 t)\right)(x-y)\right)\right)+\frac{1}{2} F(y)+A\left(2 d_{\mathbb{Z}}(t)(x-y)\right) .
\end{aligned}
$$

Using our inductive hypothesis with $2 t$ instead of $t$, it follows that

$$
\begin{aligned}
2 t F(x) & +(1-2 t) F(y)+\sum_{k=0}^{n-1} \frac{1}{2^{k}} A\left(2 d_{\mathbb{Z}}\left(2^{k}(2 t)\right)(x-y)\right) \\
& \subseteq \mathrm{cl}\left(F(2 t x+(1-2 t) y)+\frac{1}{2^{n}} H+K+\sum_{k=0}^{n-1} \frac{1}{2^{k}} B\left(2 d_{\mathbb{Z}}\left(2^{k}(2 t)\right)(x-y)\right)\right) .
\end{aligned}
$$


Combining the inclusions (45), (47) and (48), we arrive at

$$
\begin{aligned}
& t F(x)+(1-t) F(y)+\sum_{k=0}^{n} \frac{1}{2^{k}} A\left(2 d_{\mathbb{Z}}\left(2^{k} t\right)(x-y)\right) \\
& \subseteq \frac{1}{2} \operatorname{cl}\left(F(2 t x+(1-2 t) y)+\frac{1}{2^{n}} H+K+\sum_{k=0}^{n-1} \frac{1}{2^{k}} B\left(2 d_{\mathbb{Z}}\left(2^{k}(2 t)\right)(x-y)\right)\right)+\frac{1}{2} F(y)+A\left(2 d_{\mathbb{Z}}(t)(x-y)\right) \\
& \subseteq \operatorname{cl}\left(\frac{F(2 t x+(1-2 t) y)+F(y)}{2}+\frac{1}{2^{n+1}} H+K+\sum_{k=0}^{n-1} \frac{1}{2^{k+1}} B\left(2 d_{\mathbb{Z}}\left(2^{k}(2 t)\right)(x-y)\right)+A(2 t(x-y))\right)
\end{aligned}
$$

By the Jensen-convexity property (28) of $F$, we have that

$$
\frac{F(2 t x+(1-2 t) y)+F(y)}{2}+A(2 t(x-y)) \subseteq \mathrm{cl}\left(F\left(\frac{2 t x+(1-2 t) y+y}{2}\right)+B(2 t(x-y))\right) .
$$

This and the previous inclusion imply

$$
\begin{aligned}
& t F(x)+(1-t) F(y)+\sum_{k=0}^{n} \frac{1}{2^{k}} A\left(2 d_{\mathbb{Z}}\left(2^{k} t\right)(x-y)\right) \\
& \subseteq \operatorname{cl}\left(\operatorname{cl}\left(F\left(\frac{2 t x+(1-2 t) y+y}{2}\right)+B(2 t(x-y))\right)+\frac{1}{2^{n+1}} H+K+\sum_{k=0}^{n-1} \frac{1}{2^{k+1}} B\left(2 d_{\mathbb{Z}}\left(2^{k}(2 t)\right)(x-y)\right)\right) \\
& =\operatorname{cl}\left(F(t x+(1-t) y)+\frac{1}{2^{n+1}} H+K+\sum_{k=0}^{n} \frac{1}{2^{k}} B\left(2 d_{\mathbb{Z}}\left(2^{k} t\right)(x-y)\right)\right)
\end{aligned}
$$

Now, we can conclude that inclusion (40) holds for all $n \geq 0$.

To complete the proof of the theorem, let $t \in[0,1]$ be also fixed and apply Lemma 2.2 to the sequences of sets and numbers defined for $n \geq 0$ as

$$
\begin{aligned}
& A_{n}:=t F(x)+(1-t) F(y)+\sum_{k=0}^{n-1} \frac{1}{2^{k}} A\left(2 d_{\mathbb{Z}}\left(2^{k} t\right)(x-y)\right), \\
& B_{n}:=F(t x+(1-t) y)+\sum_{k=0}^{n-1} \frac{1}{2^{k}} B\left(2 d_{\mathbb{Z}}\left(2^{k} t\right)(x-y)\right), \\
& \varepsilon_{n}:=\frac{1}{2^{n}}
\end{aligned}
$$

Then, with these notations, inclusion (40) is equivalent to (14). On the other hand, by the assumption that $0 \in A(u) \cap B(u)$ for all $u \in(D-D)$, it easily follows that $\left(A_{n}\right)$ and $\left(B_{n}\right)$ are nondecreasing sequences of subsets of $Y$.

We will show that $K \subseteq \bigcap_{n=0}^{\infty} \overline{\operatorname{rec}}\left(B_{n}\right)$. For this, it suffices to prove, that for all neighborhood $U$ of zero and for all $n \in \mathbb{N}$, the inclusion $K \subseteq U+\operatorname{rec}\left(B_{n}\right)$ is valid. Let $n \in \mathbb{N}$ and let $U$ be an arbitrary neighborhood of zero. Then choose a neighborhood $V$ of zero such that $\sum_{k=0}^{n-1} 2^{-k} V \subseteq U$. Then, $K \subseteq V+\operatorname{rec}(B(u))$ for all $u \in D-D$. Hence, using the properties of the recession cones established 
in Lemma 2.1, we obtain

$$
\begin{aligned}
K=\sum_{k=0}^{n-1} \frac{1}{2^{k}} K & \subseteq \sum_{k=0}^{n-1} \frac{1}{2^{k}}\left(V+\operatorname{rec}\left(B\left(2 d_{\mathbb{Z}}\left(2^{k} t\right)(x-y)\right)\right)\right) \\
& \subseteq U+\operatorname{rec}\left(\sum_{k=0}^{n-1} \frac{1}{2^{k}}\left(B\left(2 d_{\mathbb{Z}}\left(2^{k} t\right)(x-y)\right)\right)\right. \\
& \subseteq U+\operatorname{rec}\left(F(t x+(1-t) y)+\sum_{k=0}^{n-1} \frac{1}{2^{k}}\left(B\left(2 d_{\mathbb{Z}}\left(2^{k} t\right)(x-y)\right)\right),\right.
\end{aligned}
$$

which proves the inclusion $K \subseteq U+\operatorname{rec}\left(B_{n}\right)$. Therefore $K \subseteq \bigcap_{n=0}^{\infty} \overline{\operatorname{rec}}\left(B_{n}\right)$.

Now we are in the position to apply Lemma 2.2. Hence (15) holds, in other words, we obtain that

$$
\begin{aligned}
\mathrm{cl}\left(\bigcup_{n=1}^{\infty}(t F(x)+(1-t) F(y)\right. & \left.\left.+\sum_{k=0}^{n-1} \frac{1}{2^{k}} A\left(2 d_{\mathbb{Z}}\left(2^{k} t\right)(x-y)\right)\right)\right) \\
& \subseteq \operatorname{cl}\left(\bigcup_{n=1}^{\infty}\left(F(t x+(1-t) y)+\sum_{k=0}^{n-1} \frac{1}{2^{k}} B\left(2 d_{\mathbb{Z}}\left(2^{k} t\right)(x-y)\right)\right)\right) .
\end{aligned}
$$

Now applying the equality in (13) to evaluate the left and right hand sides of the above inclusion, it follows that

$$
\begin{aligned}
\operatorname{cl}(t F(x)+(1-t) F(y) & \left.+\mathrm{cl}\left(\bigcup_{n=1}^{\infty} \sum_{k=0}^{n-1} \frac{1}{2^{k}} A\left(2 d_{\mathbb{Z}}\left(2^{k} t\right)(x-y)\right)\right)\right) \\
& \subseteq \mathrm{cl}\left(F(t x+(1-t) y)+\mathrm{cl}\left(\bigcup_{n=1}^{\infty} \sum_{k=0}^{n-1} \frac{1}{2^{k}} B\left(2 d_{\mathbb{Z}}\left(2^{k} t\right)(x-y)\right)\right)\right),
\end{aligned}
$$

which is equivalent to the inclusion (29) to be proved.

Proof of Theorem 4.2. To prove (31), we are going to show first that, for all $x, y \in D$, there exists a bounded set $H \subseteq Y$ such that, for all $n \geq 0$ and $t \in[0,1]$,

$$
\begin{aligned}
F(t x+(1-t) y) & +\sum_{k=0}^{n-1} \frac{1}{2^{k}} A\left(2 d_{\mathbb{Z}}\left(2^{k} t\right)(x-y)\right) \\
& \subseteq \mathrm{cl}\left(t F(x)+(1-t) F(y)+\frac{1}{2^{n}} H+K+\sum_{k=0}^{n-1} \frac{1}{2^{k}} B\left(2 d_{\mathbb{Z}}\left(2^{k} t\right)(x-y)\right)\right) .
\end{aligned}
$$

Let $x, y \in D$ be fixed. To verify that (49) holds, we will proceed by induction over $n$.

The bounded set $H$ will be constructed so that (49) be valid for $n=0$, that is, for all $t \in[0,1]$, the following condition holds:

$$
F(t x+(1-t) y) \subseteq \mathrm{cl}(t F(x)+(1-t) F(y)+H+K) .
$$

In view of Lemma 2.3, the local closed $K$-lower boundedness of $F$ and the compactness of the segment $[x, y]$ imply that there exists a bounded set $H_{0} \subseteq Y$ such that

$$
F(t x+(1-t) y) \subseteq \operatorname{cl}\left(H_{0}+K\right) \quad(t \in[0,1]) .
$$


On the other hand, the sets $F(x)$ and $F(y)$ being nonempty, we can choose two elements $u \in F(x)$ and $v \in F(y)$. Then

$$
0 \in F(x)-u \quad \text { and } \quad 0 \in F(y)-v \text {. }
$$

Multiplying the two inclusions in (52) by $t$ and $(1-t)$, respectively, and adding them up to the inclusion (51), for $t \in[0,1]$, we obtain that

$$
\begin{aligned}
F(t x+(1-t) y) & \subseteq t F(x)+(1-t) F(y)-t u-(1-t) v+\operatorname{cl}\left(H_{0}+K\right) \\
& \subseteq \operatorname{cl}\left(t F(x)+(1-t) F(y)-[u, v]+H_{0}+K\right) .
\end{aligned}
$$

Therefore, (50) holds with $H:=H_{0}-[u, v]$, which is obviously bounded.

Now, suppose that (49) is valid for $n$ and let us prove its validity for $n+1$. Assume that $t \in\left[0, \frac{1}{2}\right]$. Observe that then $d_{\mathbb{Z}}(t)=t$. Let us start evaluating the left side of the inclusion to be proved.

$$
\begin{aligned}
F(t x & +(1-t) y)+\sum_{k=0}^{n} \frac{1}{2^{k}} A\left(2 d_{\mathbb{Z}}\left(2^{k} t\right)(x-y)\right) \\
& =F(t x+(1-t) y)+A\left(2 d_{\mathbb{Z}}(t)(x-y)\right)+\sum_{k=1}^{n} \frac{1}{2^{k}} A\left(2 d_{\mathbb{Z}}\left(2^{k} t\right)(x-y)\right) \\
& =F\left(\frac{2 t x+(1-2 t) y+y}{2}\right)+A(2 t(x-y))+\frac{1}{2} \sum_{k=0}^{n-1} \frac{1}{2^{k}} A\left(2 d_{\mathbb{Z}}\left(2^{k}(2 t)\right)(x-y)\right) .
\end{aligned}
$$

By the Jensen-concavity property (30) of $F$, we get the inclusion

$$
F\left(\frac{2 t x+(1-2 t) y+y}{2}\right)+A(2 t(x-y)) \subseteq \mathrm{cl}\left(\frac{F(2 t x+(1-2 t) y)+F(y)}{2}+B(2 t(x-y))\right) .
$$

Combining (53) and (54), we obtain

$$
\begin{aligned}
& F(t x+(1-t) y)+\sum_{k=0}^{n} \frac{1}{2^{k}} A\left(2 d_{\mathbb{Z}}\left(2^{k} t\right)(x-y)\right) \\
& \quad \subseteq \mathrm{cl}\left(\frac{F(2 t x+(1-2 t) y)+F(y)}{2}+B(2 t(x-y))\right)+\frac{1}{2} \sum_{k=0}^{n-1} \frac{1}{2^{k}} A\left(2 d_{\mathbb{Z}}\left(2^{k}(2 t)\right)(x-y)\right) \\
& \quad \subseteq \operatorname{cl}\left(\frac{1}{2}\left(F(2 t x+(1-2 t) y)+\sum_{k=0}^{n-1} \frac{1}{2^{k}} A\left(2 d_{\mathbb{Z}}\left(2^{k}(2 t)\right)(x-y)\right)\right)+\frac{1}{2} F(y)+B\left(2 d_{\mathbb{Z}}(t)(x-y)\right)\right) .
\end{aligned}
$$

Now, using our inductive hypothesis with $2 t$ instead of $t$, we get

$$
\begin{aligned}
F(2 t x & +(1-2 t) y)+\sum_{k=0}^{n-1} \frac{1}{2^{k}} A\left(2 d_{\mathbb{Z}}\left(2^{k}(2 t)\right)(x-y)\right) \\
& \subseteq \operatorname{cl}\left(2 t F(x)+(1-2 t) F(y)+\frac{1}{2^{n}} H+K+\sum_{k=0}^{n-1} \frac{1}{2^{k}} B\left(2 d_{\mathbb{Z}}\left(2^{k}(2 t)\right)(x-y)\right)\right) .
\end{aligned}
$$


Inserting (56) into (55) and using that $(1-2 t) F(y)+F(y) \subseteq \operatorname{cl}((2-2 t) F(y)+K)$ (which is a consequence of the pointwise closed $K$-convexity of $F)$, it follows that

$$
\begin{aligned}
& F(t x+(1-t) y)+\sum_{k=0}^{n} \frac{1}{2^{k}} A\left(2 d_{\mathbb{Z}}\left(2^{k} t\right)(x-y)\right) \\
& \subseteq \mathrm{cl}\left(\frac{1}{2} \mathrm{cl}\left(2 t F(x)+(1-2 t) F(y)+\frac{1}{2^{n}} H+K+\sum_{k=0}^{n-1} \frac{1}{2^{k}} B\left(2 d_{\mathbb{Z}}\left(2^{k}(2 t)\right)(x-y)\right)\right)\right. \\
& \left.+\frac{1}{2} F(y)+B\left(2 d_{\mathbb{Z}}(t)(x-y)\right)\right) \\
& \subseteq \mathrm{cl}\left(\frac{1}{2}(2 t F(x)+(1-2 t) F(y)+F(y)+K)+\frac{1}{2^{n+1}} H+\sum_{k=0}^{n} \frac{1}{2^{k}} B\left(2 d_{\mathbb{Z}}\left(2^{k} t\right)(x-y)\right)\right) \\
& \subseteq \mathrm{cl}\left(t F(x)+(1-t) F(y)+\frac{1}{2^{n+1}} H+K+\sum_{k=0}^{n} \frac{1}{2^{k}} B\left(2 d_{\mathbb{Z}}\left(2^{k} t\right)(x-y)\right)\right) .
\end{aligned}
$$

This completes the proof of the induction and hence (49) holds for all $n \geq 0$.

Now we are going to use Lemma 2.2 , so that, for a fixed $t \in[0,1]$, we define the sequences

$$
\begin{aligned}
& A_{n}:=F(t x+(1-t) y)+\sum_{k=0}^{n-1} \frac{1}{2^{k}} A\left(2 d_{\mathbb{Z}}\left(2^{k} t\right)(x-y)\right), \\
& B_{n}:=t F(x)+(1-t) F(y)+\sum_{k=0}^{n-1} \frac{1}{2^{k}} B\left(2 d_{\mathbb{Z}}\left(2^{k} t\right)(x-y)\right), \\
& \varepsilon_{n}=\frac{1}{2^{n}} .
\end{aligned}
$$

Then, inclusion (49), with sequences $\left(A_{n}\right),\left(B_{n}\right)$ and $\left(\varepsilon_{n}\right)$ defined above is equivalent to (14). One can see that the sequences $\left(A_{n}\right)$ and $\left(B_{n}\right)$ are nondecreasing and $K \subseteq \bigcap_{n=0}^{\infty} \frac{\overline{r e c}}{\operatorname{re}}\left(B_{n}\right)$ also holds (due to a similar argument that was followed in the proof of Theorem 4.1). Thus, by the Lemma 2.2, it follows that

$$
\begin{aligned}
\operatorname{cl}\left(\bigcup_{n=0}^{\infty} F(t x+(1-t) y)\right. & \left.+\sum_{k=0}^{n-1} \frac{1}{2^{k}} A\left(2 d_{\mathbb{Z}}\left(2^{k} t\right)(x-y)\right)\right) \\
& \subseteq \mathrm{cl}\left(\bigcup_{n=0}^{\infty} t F(x)+(1-t) F(y)+\sum_{k=0}^{n-1} \frac{1}{2^{k}} B\left(2 d_{\mathbb{Z}}\left(2^{k} t\right)(x-y)\right)\right) .
\end{aligned}
$$

Now, similarly as in the proof of Theorem 4.1, using (13), this relation implies the desired inclusion (31).

\section{REFERENCES}

[1] A. Averna and T. Cardinali, On the concepts of $K$-convexity [K-concavity] and $K$-convexity* $\left[K\right.$-concavity $\left.{ }^{*}\right]$, Riv. Mat. Univ. Parma (4) 16 (1990), no. 1-2, 311-330. MR 1105752 (92h:26031)

[2] A. Azócar, J. Giménez, K. Nikodem, and J. L. Sánchez, On strongly midconvex functions, Opuscula Math. 31 (2011), no. 1, 15-26. MR 2739838 (2011k:26009)

[3] F. Bernstein and G. Doetsch, Zur Theorie der konvexen Funktionen, Math. Ann. 76 (1915), no. 4, 514-526. MR 1511840 
[4] Z. Boros, An inequality for the Takagi function, Math. Inequal. Appl. 11 (2008), no. 4, 757-765. MR 2009f:39047

[5] J.M. Borwein, Multivalued convexity and optimization: a unified approach to inequality and equality constraints, Math. Programming 13 (1977), no. 2, 183-199. MR 0451166 (56 \#9453)

[6] T. Cardinali, K. Nikodem, and F. Papalini, Some results on stability and on characterization of K-convexity of set-valued functions, Ann. Polon. Math. 58 (1993), no. 2, 185-192. MR 1239022 (94g:26022)

[7] A. Házy, On approximate t-convexity, Math. Inequal. Appl. 8 (2005), no. 3, 389-402. MR 2148233 (2006c:26019)

[8] A. Házy, On the stability of t-convex functions, Aequationes Math. 74 (2007), no. 3, 210-218. MR 2376448 (2008j:26012)

[9] A. Házy and Zs. Páles, On approximately midconvex functions, Bull. London Math. Soc. 36 (2004), no. 3, 339-350. MR 2038721 (2004j:26020)

[10] A. Házy and Zs. Páles, On approximately t-convex functions, Publ. Math. Debrecen 66 (2005), no. 3-4, 489-501. MR 2137784 (2006c:26023)

[11] A. Házy and Zs. Páles, On a certain stability of the Hermite-Hadamard inequality, Proc. R. Soc. Lond. Ser. A Math. Phys. Eng. Sci. 465 (2009), no. 2102, 571-583. MR 2471774 (2009k:39033)

[12] M. Kuczma, An Introduction to the Theory of Functional Equations and Inequalities, Prace Naukowe Uniwersytetu Śląskiego w Katowicach, vol. 489, Państwowe Wydawnictwo Naukowe - Uniwersytet Śląski, Warszawa-Kraków-Katowice, 1985, 2nd edn. (ed. by A. Gilányi), Birkhäuser, Basel, 2009. MR 0788497 (86i:39008), MR 2467621

[13] M. Laczkovich, The local stability of convexity, affinity and of the Jensen equation, Aequationes Math. 58 (1999), 135-142. MR 1714327 (2001d:39028)

[14] H. Leiva, N. Merentes, K. Nikodem, and J. L. Sánchez, Strongly convex set-valued maps, J. Global Optim. 57 (2013), 695-705. MR 3119375

[15] J. Makó and Zs. Páles, Approximate convexity of Takagi type functions, J. Math. Anal. Appl. 369 (2010), no. 2, 545-554. MR 2651700 (2011k:26011)

[16] J. Makó and Zs. Páles, Implications between approximate convexity properties and approximate Hermite-Hadamard inequalities, Cent. Eur. J. Math. 10 (2012), no. 3, 1017-1041. MR 2902231

[17] J. Makó and Zs. Páles, Korovkin type theorems and approximate Hermite-Hadamard inequalities, J. Approx. Theory 164 (2012), no. 8, 1111-1142. MR 2935448

[18] J. Makó and Zs. Páles, On approximately convex Takagi type functions, Proc. Amer. Math. Soc. 141 (2013), no. 6, 2069-2080. MR 3034432

[19] A. Mureńko, Ja. Tabor, and Jó. Tabor, Applications of de Rham Theorem in approximate midconvexity, J. Diff. Equat. Appl. 18 (2012), no. 3, 335-344. MR 2901825

[20] C. T. Ng and K. Nikodem, On approximately convex functions, Proc. Amer. Math. Soc. 118 (1993), no. 1, $103-108$. MR 1159176 (93f:26006)

[21] K. Nikodem, Continuity of K-convex set-valued functions, Bull. Polish Acad. Sci. Math. 34 (1986), no. 7-8, 393-400. MR 874882 (88a:26040)

[22] K. Nikodem, On concave and midpoint concave set-valued functions, Glas. Mat. Ser. III 22(42) (1987), no. 1, 69-76. MR 940094 (89g:39017)

[23] K. Nikodem, On midpoint convex set-valued functions, Aequationes Math. 33 (1987), no. 1, 46-56. MR 901799 (88h:90171)

[24] K. Nikodem, K-convex and K-concave set-valued functions, Zeszyty Nauk. Politech. Łódz. Mat. (Łódz) 559 (1989), 1-75, (Rozprawy Nauk. 114).

[25] F. Papalini, The K-midpoint * convexity [concavity] and lower [upper] K-semicontinuity of a multifunction, Riv. Mat. Univ. Parma (4) 16 (1990), no. 1-2, 149-159 (1991). MR 1105736 (92h:26032)

[26] B. T. Polyak, Existence theorems and convergence of minimizing sequences for extremal problems with constraints, Dokl. Akad. Nauk SSSR 166 (1966), 287-290. MR 33 \#6466

[27] W. Rudin, Functional Analysis, second ed., International Series in Pure and Applied Mathematics, McGraw-Hill Inc., New York, 1991. MR 1157815 (92k:46001)

[28] H. Rådström, One-parameter semigroups of subsets of a real linear space, Ark. Mat. 4 (1960), 87-97. MR 0146280 $(26 \# 3802)$

[29] Ja. Tabor and Jó. Tabor, Generalized approximate midconvexity, Control Cybernet. 38 (2009), no. 3, 655-669. MR 2650358 (2011f:52002) 
[30] Ja. Tabor and Jó. Tabor, Takagi functions and approximate midconvexity, J. Math. Anal. Appl. 356 (2009), no. 2, 729-737.

[31] Ja. Tabor, Jó. Tabor, and M. Żołdak, Approximately convex functions on topological vector spaces, Publ. Math. Debrecen 77 (2010), 115-123. MR 2675738 (2011f:26009)

[32] Ja. Tabor, Jó. Tabor, and M. Żołdak, Optimality estimations for approximately midconvex functions, Aequationes Math. 80 (2010), 227-237. MR 2736954 (2011j:26020)

[33] L. I. Trudzik, Continuity properties of vector-valued convex functions, J. Austral. Math. Soc. Ser. A 36 (1984), no. 3, 404-415. MR 733912 (85d:46062)

Escuela de Matematicas, Universidad Central de Venezuela, Caracas, Venezuela

E-mail address: carlosl.gonzalez@ciens.ucv.ve

Department of Mathematics and Computer Science, University of Bielsko-Biala, Ul. Willowa 2, 43-309 Bielsko-Biata, Poland

E-mail address: knikodem@ath.bielsko.pl

Institute of Mathematics, University of Debrecen, H-4010 Debrecen, Pf. 12, Hungary

E-mail address: pales@science.unideb.hu

Escuela de Matematicas, Universidad Central de Venezuela, Caracas, Venezuela

E-mail address: gariroa@gmail.com 